\title{
The Study of Fractional Order Controller with SLAM in the Humanoid Robot
}

\author{
Shuhuan Wen, ${ }^{1}$ Xiao Chen, ${ }^{1}$ Yongsheng Zhao, ${ }^{2}$ Ahmad B. Rad, \\ Kamal Mohammed Othman, ${ }^{3}$ and Ethan Zhang $^{3}$ \\ ${ }^{1}$ Key Lab of Industrial Computer Control Engineering of Hebei Province, Yanshan University, \\ Qinhuangdao 066004, China \\ ${ }^{2}$ Machanical Engineering, Yanshan University, Qinhuangdao 066004, China \\ ${ }^{3}$ School of Engineering Sciences, Simon Fraser University, 250-13450, 102 Avenue, \\ Surrey, BC, Canada V3T 0A3
}

Correspondence should be addressed to Yongsheng Zhao; yszhao@ysu.edu.cn

Received 26 September 2013; Revised 15 November 2013; Accepted 17 November 2013; Published 28 January 2014

Academic Editor: Ming Li

Copyright (C) 2014 Shuhuan Wen et al. This is an open access article distributed under the Creative Commons Attribution License, which permits unrestricted use, distribution, and reproduction in any medium, provided the original work is properly cited.

\begin{abstract}
We present a fractional order PI controller (FOPI) with SLAM method, and the proposed method is used in the simulation of navigation of NAO humanoid robot from Aldebaran. We can discretize the transfer function by the Al-Alaoui generating function and then get the FOPI controller by Power Series Expansion (PSE). FOPI can be used as a correction part to reduce the accumulated error of SLAM. In the FOPI controller, the parameters $\left(K_{p}, K_{i}\right.$, and $\left.\alpha\right)$ need to be tuned to obtain the best performance. Finally, we compare the results of position without controller and with PI controller, FOPI controller. The simulations show that the FOPI controller can reduce the error between the real position and estimated position. The proposed method is efficient and reliable for NAO navigation.
\end{abstract}

\section{Introduction}

More than $95 \%$ of the control loops are of PI/PID type in process control, but only a few of control loops work well [1]. There are some problems in PI/PID control, such as bad tuning, incorrect implementation techniques, some restrictions and limitations, and actuator and sensor problems.

Using the notion of fractional order, it may be a step closer to the real world life, because many real physical systems are well characterized by fractional order differential equations [2]. So a considerable amount of research in fractional order dynamic systems and controllers was published in scientific and engineering literature in the last few years. Modern examples of applications are bioengineering [3], physics [4], chaos theory [5], viscoelasticity [6], and many others (see, e.g., [7]). Fractional order controllers can be expressed by fractional order differential equations. Fractional order controllers have been used in industrial applications [8] and various fields such as power electronics [9], system identification [10], robotic manipulators, irrigation canal control [11], mechatronics systems [12], and heat diffusion systems [13]. In addition, the stability is an essential property of systems, for example, for the stability of conventional systems of integer order and for fractional systems. So the stability issues for fractional order have been solved [2, 14-19].

In this paper, we will study fractional order PI (FOPI) controller combined with SLAM in the navigation of humanoid robot because of the limitation of PI controller. In the FOPI controller, the parameters $\left(K_{p}, K_{i}\right.$, and $\left.\alpha\right)$ need to be tuned based on some design specifications. The results will be compared without controller and with PI controller, fractional order PI controller.

The rest of this paper is organized as follows: the FOPI controller used in the NAO robot navigation control is presented in Section 2, the background knowledge of SLAM and the NAO robot are described in Section 3, and the simulation results and conclusion are given in Sections 4 and 5 , respectively. 


\section{Fractional Order PI Controller}

2.1. Fractional Calculus. The fractional order $P I^{\lambda} D^{u}$ was proposed as a generalization of the PID controller [20]. A general fundamental operator is denoted as follows:

$$
{ }_{a} D_{t}^{\alpha}= \begin{cases}\frac{d^{\alpha}}{d t^{\alpha}} & \operatorname{Re}(\alpha)>0 \\ 1 & \operatorname{Re}(\alpha)=0 \\ \int_{a}^{t}(d \tau)^{-\alpha}, & \operatorname{Re}(\alpha)<0\end{cases}
$$

where $a$ and $t$ are the limit of the operation, $\alpha$ is the fractional order, which can be any complex number, and $\tau$ is the variable of integration.

Commonly, there are three methods to define the fractional calculus [21].

\subsubsection{Grünwald-Letnikov Definition. Consider}

$$
{ }_{a} D_{t}^{\alpha} f(t)=\lim _{h \rightarrow 0} \frac{1}{h^{\alpha}} \sum_{j=0}^{[(t-a) / h]}(-1)^{j}\left(\begin{array}{c}
\alpha \\
j
\end{array}\right) f(t-j h),
$$

where $\left(\begin{array}{l}\alpha \\ j\end{array}\right)=\Gamma(\alpha+1) / \Gamma(j+1) \Gamma(\alpha-j+1)=\alpha ! / j !(\alpha-j) !$ is the binomial coefficient, [.] is the integer part, $h$ is the calculation of step length, and $\Gamma(\bullet)$ is gamma function.

\subsubsection{Riemann-Liouville Definition. Consider}

$$
\begin{array}{r}
{ }_{a} D_{t}^{\alpha} f(t)=\frac{1}{\Gamma(n-\alpha)} \frac{d^{n}}{d t^{n}} \int_{a}^{t} \frac{f(\tau)}{(t-\tau)^{\alpha-n+1}} d \tau, \\
(n-1<\alpha<n) .
\end{array}
$$

\subsubsection{Caputo Definition. Consider}

$$
{ }_{a} D_{t}^{\alpha} f(t)=\frac{1}{\Gamma(n-\alpha)} \int_{a}^{t} \frac{f^{(n)}(\tau)}{(t-\tau)^{\alpha-n+1}} d \tau, \quad(n-1<\alpha<n) .
$$

For zero initial conditions and lower limit $a=0$, the Laplace transform of fractional derivatives is

$$
L\left\{{ }_{0} D_{t}^{\alpha} f(t)\right\}=s^{\alpha} F(s) .
$$

2.2. Fractional Order Controller. From the definition, it is shown that the fractional order differential is different from the integer order differential. It is not to obtain the limit values at the point, but it is related to the function values from the initial time to this point time. So the fractional order PI has memory.

The typical $n$-term linear fractional order differential equation as is follows:

$$
\begin{aligned}
a_{n} D^{\beta_{n}} y(t)+a_{n-1} D^{\beta_{n-1}} y(t)+\cdots a_{1} D^{\beta_{1}} y(t)+a_{0} D^{\beta_{0}} y(t) \\
=b_{n} D^{\alpha_{n}} u(t)+b_{n-1} D^{\alpha_{n-1}} u(t)+\cdots b_{1} D^{\alpha_{1}} u(t) \\
\quad+b_{0} D^{\alpha_{0}} u(t),
\end{aligned}
$$

where $\alpha_{k}$ and $\beta_{k}$ are arbitrary real numbers. Assuming $\alpha_{k}>$ $\alpha_{k-1}>\cdots \alpha_{0}>0, \beta_{k}>\beta_{k-1}>\cdots \beta_{0}>0$ because of no loss of generality.

Assume (6) meets the zero initial condition; we can get the continuous fractional order transfer function by using Laplace transform technique:

$$
G(s)=\frac{Y(s)}{U(s)}=\frac{b_{m} s^{\beta_{m}}+b_{m-1} s^{\beta_{m-1}}+\cdots+b_{0} s^{\beta_{0}}}{a_{n} s^{\alpha_{n}}+a_{n-1} s^{\alpha_{n}-1}+\cdots+a_{0} s^{\alpha_{0}}} .
$$

For the discrete fractional order control system, the equation $z$ transform is available:

$$
\begin{aligned}
G(z)= & \frac{Y(z)}{U(z)} \\
= & \left(b_{m}\left(w\left(z^{-1}\right)\right)^{\beta_{m}}+b_{m-1}\left(w\left(z^{-1}\right)\right)^{\beta_{m-1}}+\cdots\right. \\
& \left.\quad+b_{0}\left(w\left(z^{-1}\right)\right)^{\beta_{0}}\right) \\
& \times\left(a_{n}\left(w\left(z^{-1}\right)\right)^{\alpha_{n}}+a_{n-1}\left(w\left(z^{-1}\right)\right)^{\alpha_{n-1}}+\cdots\right. \\
& \left.\quad+a_{0}\left(w\left(z^{-1}\right)\right)^{\alpha_{0}}\right)^{-1},
\end{aligned}
$$

where $w\left(z^{-1}\right)$ is the transform function from $s$ domain to $z$ domain.

In general, the discretization of continuous fractional order differentiator/integrator $s^{ \pm \gamma}(\gamma \in R)$ can be expressed as $s^{ \pm \gamma} \approx\left(w\left(z^{-1}\right)\right)^{ \pm \gamma}$. So the generating function and its expansion determine both the form of the approximation and the coefficients [22]. We can get different generating function because of different calculation method. As a generating function, $w\left(z^{-1}\right)$, the following formula can be used in general:

$$
w\left(z^{-1}\right)=\left(\frac{1}{\mu T} \frac{1-z^{-1}}{\gamma+(1-\gamma) z^{-1}}\right)
$$

where $\mu$ and $\gamma$ denote the gain and phase tuning parameters, respectively, and $T$ is the sampling period. For example, when $\mu=1$ and $\gamma=\{0,1 / 2,7 / 8,1,3 / 2\}$, the generating function (9) becomes the forward Euler, the Tustin, the Al-Alaoui, the backward Euler, and the implicit Adams rules, respectively [23]. We will consider the Al-Alaoui generating function of the form

$$
\left(w\left(z^{-1}\right)\right)^{ \pm \gamma}=\left(\frac{8}{7 T} \frac{1-z^{-1}}{1+\left(z^{-1} / 7\right)}\right)^{ \pm \gamma} .
$$

The discretization formal for GL formula (2) is given after performing the PSE (Power Series Expansion) of (1 $\left.z^{-1}\right)^{ \pm \gamma}$. By using the short memory principle [24], the discrete equivalent of the fractional order integrodifferential operator $\left(w\left(z^{-1}\right)\right)^{ \pm \gamma}$ is given by

$$
\left(w\left(z^{-1}\right)\right)^{ \pm \gamma}=T^{\mp \gamma} z^{-[L / T]} \sum_{j=0}^{[L / T]}(-1)^{j}\left(\begin{array}{c} 
\pm \gamma \\
j
\end{array}\right) z^{[L / T]-j},
$$


where $T$ is the sampling period, $L$ is the memory length, and $(-1)^{j}\left(\begin{array}{c} \pm \gamma \\ j\end{array}\right)$ are binomial coefficients $c_{j}^{(r)}(j=0,1, \ldots), c_{0}^{(r)}=1$, and $c_{j}^{(r)}=(1-(1+( \pm \gamma)) / j) c_{j-1}^{(\gamma)}$.

We use the FOPI controller, and its transfer function can be expressed as

$$
G(s)=\frac{U(s)}{E(s)}=K_{p}+\frac{K_{i}}{s^{\alpha}}=\frac{K_{p} s^{\alpha}+K_{i}}{s^{\alpha}} .
$$

With the Al-Alaoui operation, we can obtain

$$
\begin{aligned}
G(s) & =\frac{U(s)}{E(s)}=\frac{K_{p}(8 / 7 T)^{\alpha}\left(P_{p} / Q_{q}\right)+K_{i}}{(8 / 7 T)^{\alpha}\left(P_{p} / Q_{q}\right)} \\
& =\frac{K_{p}(8 / 7 T)^{\alpha} P_{p}+K_{i} Q_{q}}{(8 / 7 T)^{\alpha} P_{p}} .
\end{aligned}
$$

The third order expansion by using PSE method is

$$
\begin{aligned}
G(s)= & \frac{U(s)}{E(s)} \\
= & \left(K_{p} K^{\alpha}\left(p_{3} z^{-3}+p_{2} z^{-2}+p_{1} z^{-1}+1\right)\right. \\
& \left.\quad+K_{i}\left(q_{3} z^{-3}+q_{2} z^{-2}+q_{1} z^{-1}+1\right)\right) \\
& \times\left(K^{\alpha}\left(p_{3} z^{-3}+p_{2} z^{-2}+p_{1} z^{-1}+1\right)\right)^{-1},
\end{aligned}
$$

where

$$
\begin{gathered}
p_{1}=-\alpha, \quad p_{2}=\frac{\alpha^{2}-\alpha}{2}, \quad p_{3}=\frac{-\alpha^{3}+3 \alpha^{2}-2 \alpha}{6}, \\
q_{1}=\frac{\alpha}{7}, \quad q_{2}=\frac{\alpha^{2}-\alpha}{98}, \quad q_{3}=\frac{\alpha^{3}-3 \alpha^{2}+2 \alpha}{2058}, \\
K^{\alpha}=\left(\frac{8}{7 T}\right)^{\alpha} .
\end{gathered}
$$

Equation (14) can be given by

$$
\begin{aligned}
G(s)= & \left(\left(K_{p} K^{\alpha} p_{3}+K_{i} q_{3}\right) z^{-3}+\left(K_{p} K^{\alpha} p_{2}+K_{i} q_{2}\right) z^{-2}\right. \\
& \left.+\left(K_{p} K^{\alpha} p_{1}+K_{i} q_{1}\right) z^{-1}+\left(K_{i}+K_{p} K^{\alpha}\right)\right) \\
& \times\left(K^{\alpha}\left(p_{3} z^{-3}+p_{2} z^{-2}+p_{1} z^{-1}+1\right)\right)^{-1} .
\end{aligned}
$$

And it can be expressed as

$$
\begin{gathered}
U(z) K^{\alpha}\left(p_{3} z^{-3}+p_{2} z^{-2}+p_{1} z^{-1}+1\right) \\
=E(z)\left[\left(K_{p} K^{\alpha} p_{3}+K_{i} q_{3}\right) z^{-3}+\left(K_{p} K^{\alpha} p_{2}+K_{i} q_{2}\right) z^{-2}\right. \\
\left.+\left(K_{p} K^{\alpha} p_{1}+K_{i} q_{1}\right) z^{-1}+\left(K_{i}+K_{p} K^{\alpha}\right)\right] .
\end{gathered}
$$

So we can get

$$
\begin{aligned}
u(t)= & \left(K_{p} p_{3}+K_{i} q_{3} K^{\alpha}\right) e(t-3) \\
& +\left(K_{p} p_{2}+K_{i} q_{2} K^{\alpha}\right) e(t-2) \\
& +\left(K_{p} p_{1}+K_{i} q_{1} K^{\alpha}\right) e(t-1) \\
& +\left(K_{p}+K_{i} K^{\alpha}\right) e(t) \\
& -p_{1} u(t-1)-p_{2} u(t-2)-p_{3} u(t-3) .
\end{aligned}
$$

\section{Background of SLAM and NAO Humanoid Robot}

The simultaneous localization and mapping (SLAM) is a process by which a robot can build a map of an environment and at the same time use this map to deduce its location. In SLAM, both the trajectory of the platform and the location of all landmarks are estimated online without the need for any a priori knowledge of location [25, 26]. EKF-SLAM is very well known to navigation problems. The main steps of SLAM include robot motion prediction, new landmarks initialization, and known landmarks correction.

The observation model is

$$
y=h(x)+v,
$$

where $x$ is robot state vector and landmark states, $h(x)$ is the observation function, and $v$ is the measurement noise.

The covariance matrix is

$$
P=F P F^{\prime}+Q
$$

where $F$ is Jacobian matrix and $Q$ is the Gaussian noise.

The EKF correction step is written as

$$
\begin{aligned}
& z=y-h\left(x^{\prime}\right), \\
& Z=H P x^{T}+R,
\end{aligned}
$$

where $z$ is the innovation's mean, $x^{\prime}$ is the observation position of the robot, $H$ is Jacobian matrix, and $R$ is the covariance matrix of the measurement noise.

Kalman gain $K$ is $K=P H^{T} Z$.

The update position of the robot is

$$
x^{\prime}=x^{\prime}+K z .
$$

The correction covariance matrix is

$$
P=P-K Z K^{T}
$$

NAO is a new biped robot recently developed by French company Aldebaran-Robotics. This humanoid robot had been designed purposely to look approachable. With a height of 0.57 meters and weight of about 4.5 kilogram, NAO has the appealing appearance of a human toddler [27]. Based on NAO robot, the FOPI controller as the correction part is used in the SLAM simulation of NAO navigation in this paper. 


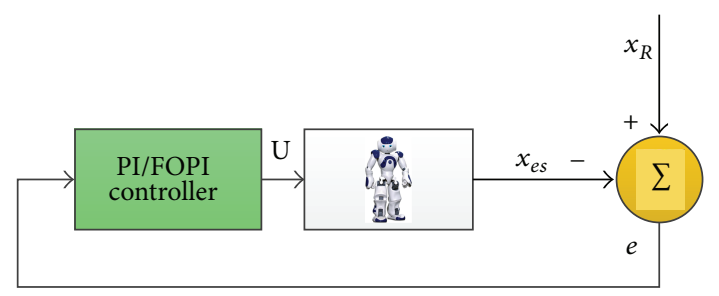

FIGURE 1: The correction part, where $x_{R}$ is the real position and $x_{e s}$ is the EKF-estimated position.

\section{Simulation Result}

4.1. Control System Model. When NAO robot moves in 2D space, the motion is mainly influenced by three variables, namely, the robot current position, the control variable, and the disturbance variable. Therefore, it is very important to reduce the accumulated error for robot and effectively overcome the negative influence of disturbance variable.

NAO will deviate from a set target because there is no correction part to compensate noise of the sensors. When the control $U$ is a fixed value, the real position and estimated position of the NAO will be largely different. So we use controller as the correction part to reduce the error. The correction part is based on the return error of the real position and estimated position of NAO. The outline of this correction part is presented in Figure 1.

4.2. Algorithm Performance Index. Because the NAO in SLAM mainly produces two kinds of errors, so the average error index of robot position, the average error index of landmarks, and the average variance index of robot position are proposed to test the performance of the controller. The average error index of robot position is the average different value between the real position and the estimated position. When NAO moves in $s$ steps, the definition $\bar{e}$ is expressed by

$$
\bar{e}=\frac{\sum_{i=1}^{s} e}{s},
$$

where $e$ is the error of $x_{R}$ and $x_{e s}$ and $s$ is the number of steps.

If the number of landmarks is $n$ and $l e$ is the error of the real landmarks and estimated landmarks, the average error index of landmarks $\overline{l e}$ is defined as follows:

$$
\overline{l e}=\frac{\sum_{i=1}^{n} l e}{n} .
$$

According to (24) and (25), we can see that the smaller the average error, the better the control effect. But it could not judge the stability of the control system from the single average error index. So we define the average variance that shows the distribution degree of error. The average variance $\sigma$ is defined as follows:

$$
\sigma=\frac{\sum_{i=1}^{s}(e-\bar{e})^{2}}{s} .
$$

The smaller the average variance, that is, every step error of $\mathrm{NAO}$ is close to the average error, the better the stability of the control.

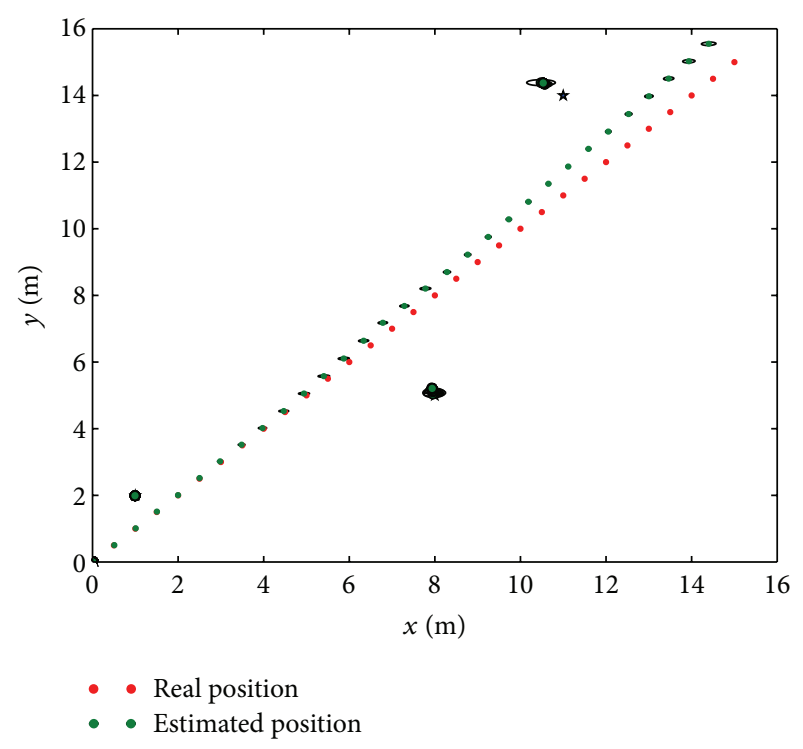

FIgURE 2: The simulation motion result of NAO in SLAM with the fixed $U$.

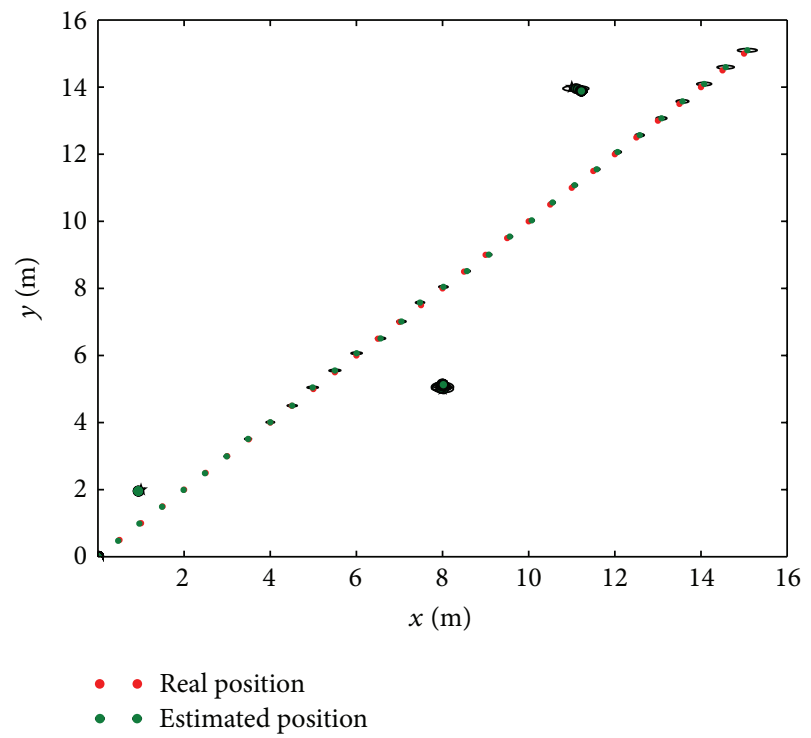

FIGURE 3: The simulation motion result of NAO in SLAM with PI controller.

4.3. Control Algorithm Comparison. Figures 2 and 3 show the simulation result of SLAM with the fixed U and PI controller, respectively. The three stars are the landmarks. The red points and green points are the real position and the estimated position, respectively. The circles on the green points are the covariance of the robot. The circles become smaller when the robot is near the landmark. The green circles are the estimated landmarks.

In this paper, if not specified, abscissa represents steps, and ordinate represents the error. Figure 4 shows the error of the different $K_{p}$ with PI controller in EKF-SLAM. When $K_{p}<$ 0.7 , the PI controller cannot effectively adjust the control variable according to the error. And when $0.7<K_{p}<1$, 


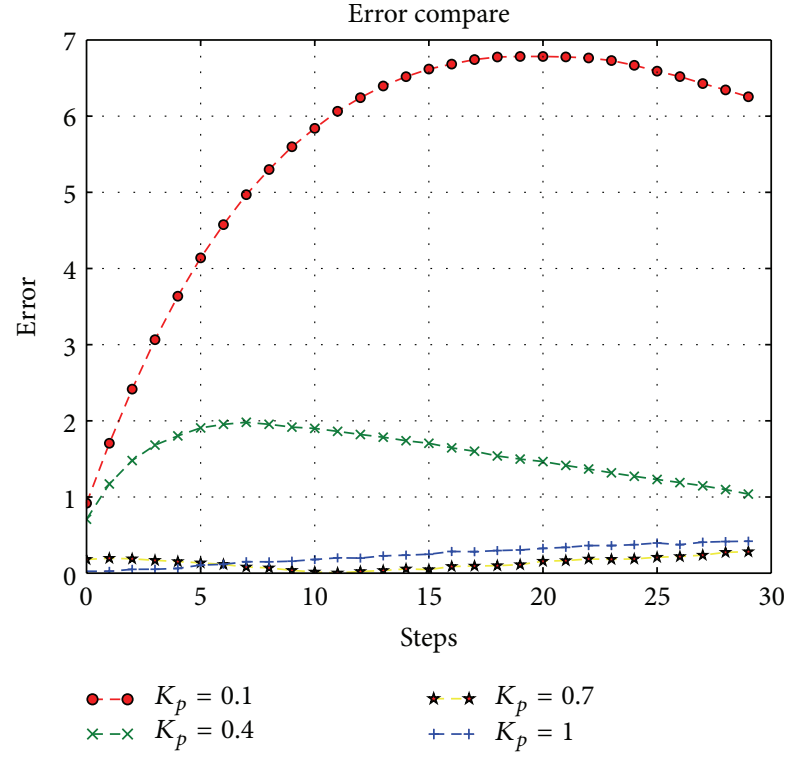

FIgURE 4: The error of the different $K_{p}$ with PI controller.

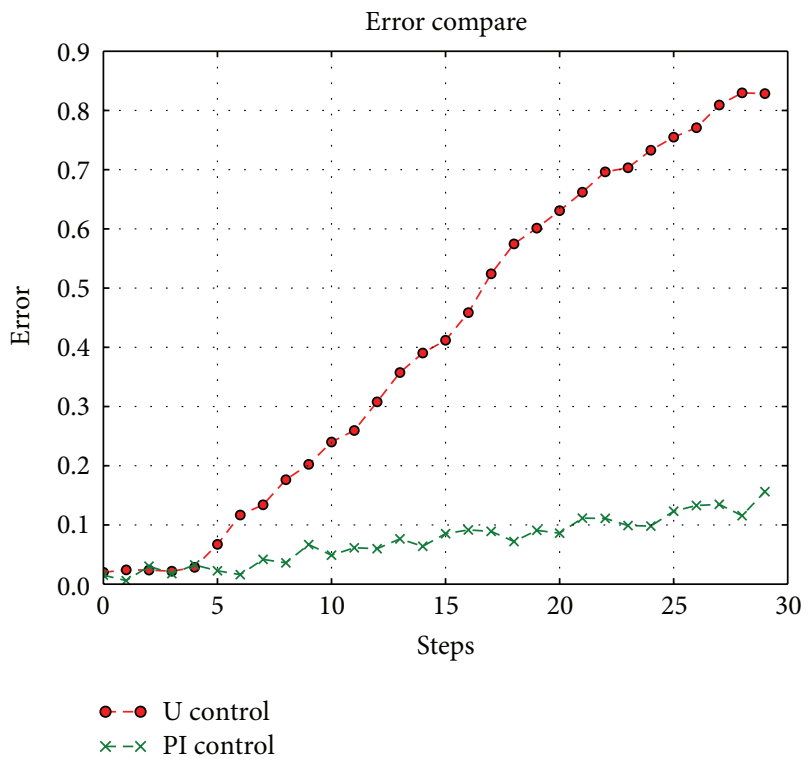

FIGURE 5: The comparison of the error with fixed U and PI controller.

the control variable generated from PI controller can basically make the estimated position trace to the real position. But when $K_{p}$ is too large, the PI control variable cannot keep stable, so we cannot follow the estimated position of robot.

Figure 5 shows the comparison of the errors with fixed $\mathrm{U}$ and PI controller. It can be seen that the error with PI controller is far less than the one with the fixed U. However, when the steps of the robot motion increase, the error generated from PI controller also gradually increases. So PI controller cannot efficiently reduce the accumulated error of the robot with the increasing steps. Therefore, we use FOPI controller to decrease the error in the motion process. There are three parameters $\left(K_{p}, K_{i}\right.$, and $\left.\alpha\right)$ to be tuned.
TABLE 1: The average error index and variance index of robot position with PI/FOPI controller.

\begin{tabular}{lcc}
\hline Controller & PI & FOPI \\
\hline $\bar{e}$ & 0.074 & 0.015 \\
$\sigma$ & 0.014 & 0.00005 \\
\hline
\end{tabular}

TABLE 2: The average error of the real landmarks and estimated landmarks with PI/FOPI controller.

\begin{tabular}{lcc}
\hline Controller & PI & FOPI \\
\hline$\overline{l e}$ & 0.167 & 0.083 \\
\hline
\end{tabular}

From Figure 6, we can know that the scope of $K_{p}$ is $0.7-0.9, K_{i}$ is about 0.2 , and $\alpha$ is the value of about 0.1 . In Figure 6(c), if the parameter $\alpha$ increases, the result of controller will become bad. But when $\alpha$ is too small, the effect of integral is not obvious, so the value of $\alpha$ is about 0.1. In the permitted range of proportional coefficient $K_{p}$ and integral coefficient $K_{i}$, the FOPI error influenced by the changing of $K_{p}$ or $K_{i}$ will not be a linear increasing trend, and its curve will move up or down with the increasing $K_{p}$ or $K_{i}$. There is a fundamental difference between FOPI controller and PI controller. The error in FOPI controller has oscillation trend, which reflects that FOPI has a better control performance.

Based on the analysis of the parameters, this paper selects the optimal parameters $\left(K_{p}=0.7, K_{i}=0.2\right.$, and $\left.\alpha=0.1\right)$ of FOPI controller. The simulation of motion result of EKFSLAM with FOPI controller is given in Figure 7. We can conclude that FOPI control can keep stable and get a better dynamics performance. The NAO robot's estimated position has almost completely been close to the real position.

Figure 8 is the comparison between the optimal parameters of FOPI controller $\left(K_{p}=0.7, K_{i}=0.2\right.$, and $\left.\alpha=0.1\right)$ and PI controller $\left(K_{p}=0.9, K_{i}=0.02\right)$. We compare them with the fixed $U$. The results verify the validity of FOPI controller. The average error and average variance are listed in Table 1. When NAO moves 0.5 each step, we can get that the error rate has got to $14 \%$ with PI controller and the error rate to approximately $3 \%$ with FOPI controller from Table 1. According to the index, it is shown that FOPI controller has better stability and higher accuracy. In addition, the average errors of the real landmarks and estimated landmarks with PI and FOPI controller are given in Table 2. We get the errors between the real landmarks and estimated landmarks based on the coordinates $[1,2,5,8,11,14]$. In order to ensure the accuracy of simulation, we do the simulation 30 times, respectively, and get the $\overline{l e}$ in Table 2 . We can see that the errors between the real landmarks and estimated landmarks based on FOPI controller with EKF-SLAM are much smaller than PI controller.

From the above figures and tables we can see that the pros and cons of three control methods are very obvious. FOPI controller is superior to PI controller, and PI controller is better than the fixed $U$ control. The error of the robot movement with FOPI controller gradually tends to zero with the increasing steps. Moreover, the error of FOPI controller will not gradually increase and remain oscillation trend 


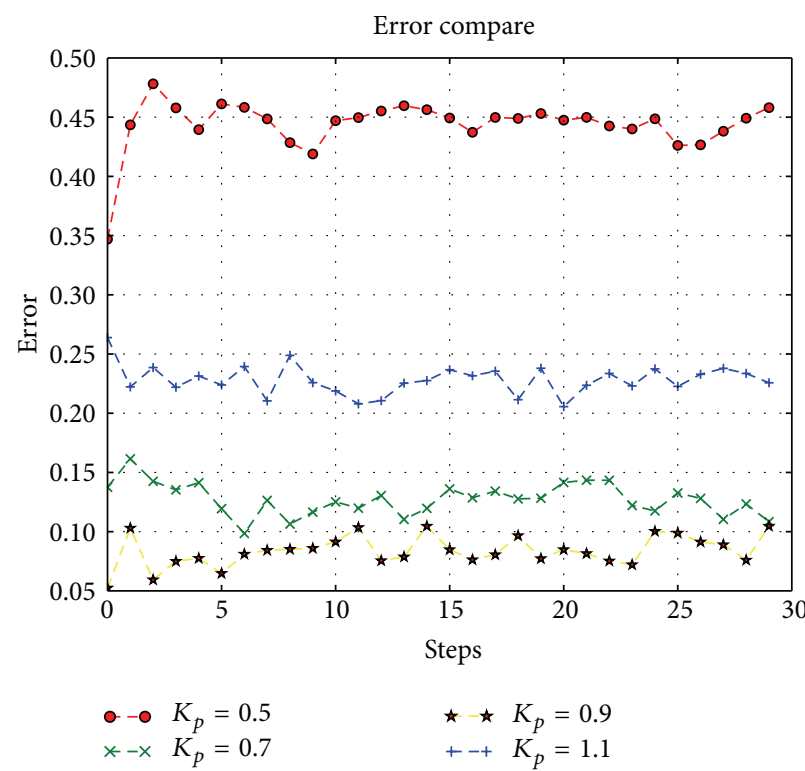

(a) The error of different $K_{p}$

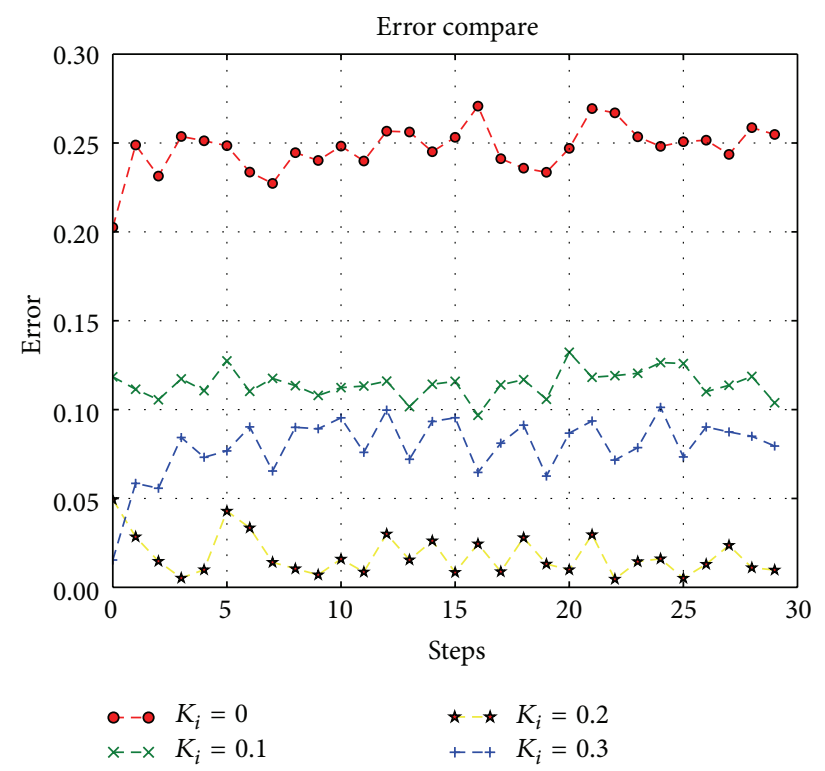

(b) The error of different $K_{i}$

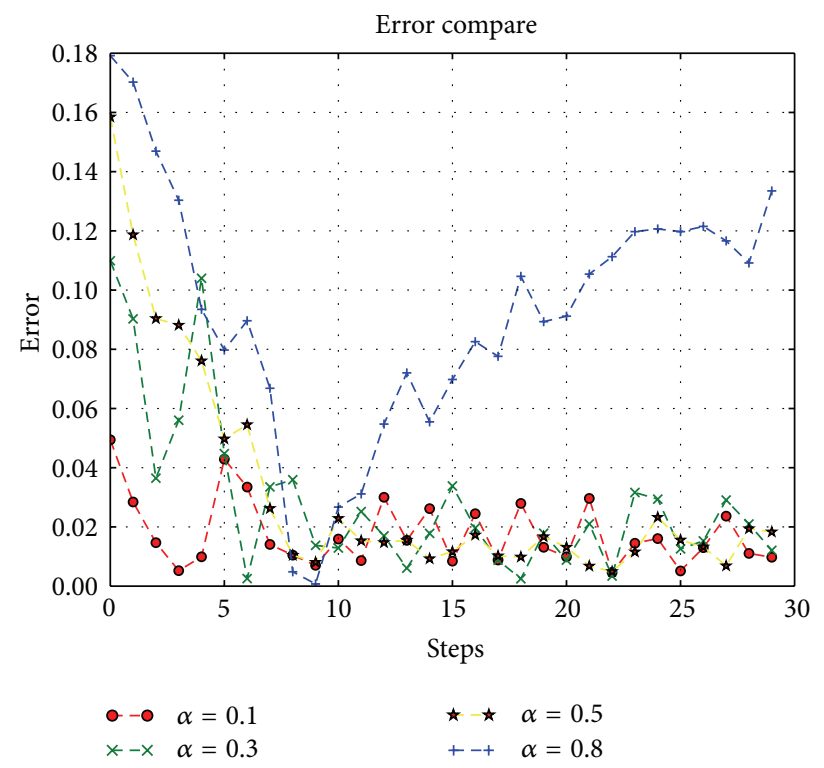

(c) The error of different $\alpha$

FIgURE 6: The effect of three parameters $\left(K_{p}, K_{i}\right.$, and $\left.\alpha\right)$ of FOPI controller.

within a certain number of steps. It is shown that FOPI has a better performance than PI.

\section{Conclusion}

In this paper, FOPI controller with EKF-SLAM is proposed. FOPI can be applied as a correction part. FOPI controller with EKF-SLAM can effectively reduce the accumulated error. The simulation shows that the FOPI controller is better than PI controller in NAO robot. The feasibility of FOPI is valid for the error of robot position and the error of landmarks. The feasibility of the proposed algorithm is validated by simulation results. In the future work, we can apply FOPI controller with SLAM to NAO platform and do the experiment in order to finish the navigation indoors environment. In addition, it is worth studying how to generalize the twodimensional EKF-SLAM problem to the multidimensional EKF-SLAM problem.

\section{Conflict of Interests}

The authors declare that there is no conflict of interests regarding the publication of this paper. 


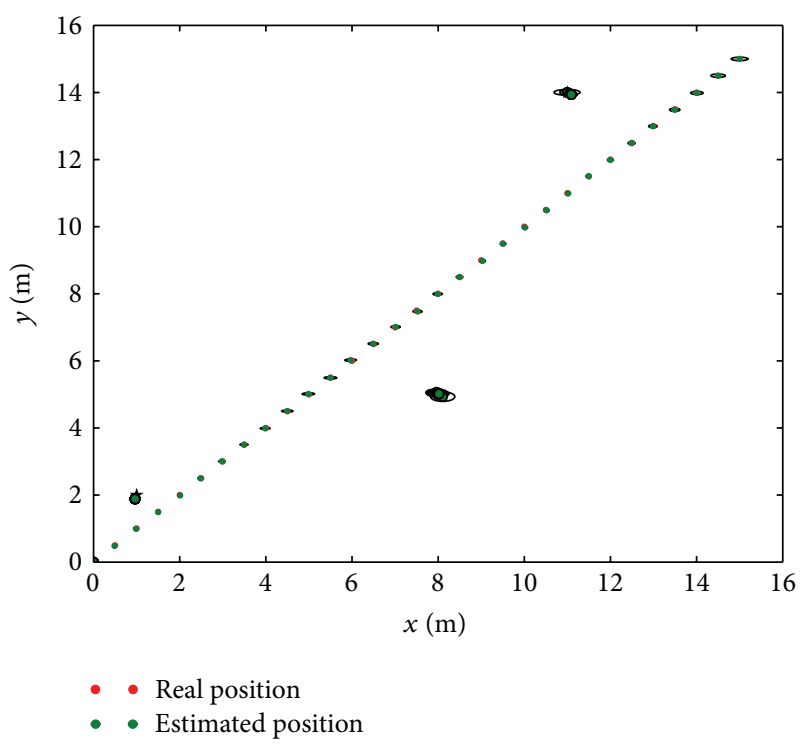

FIGURE 7: The simulation motion result of SLAM with FOPI controller.

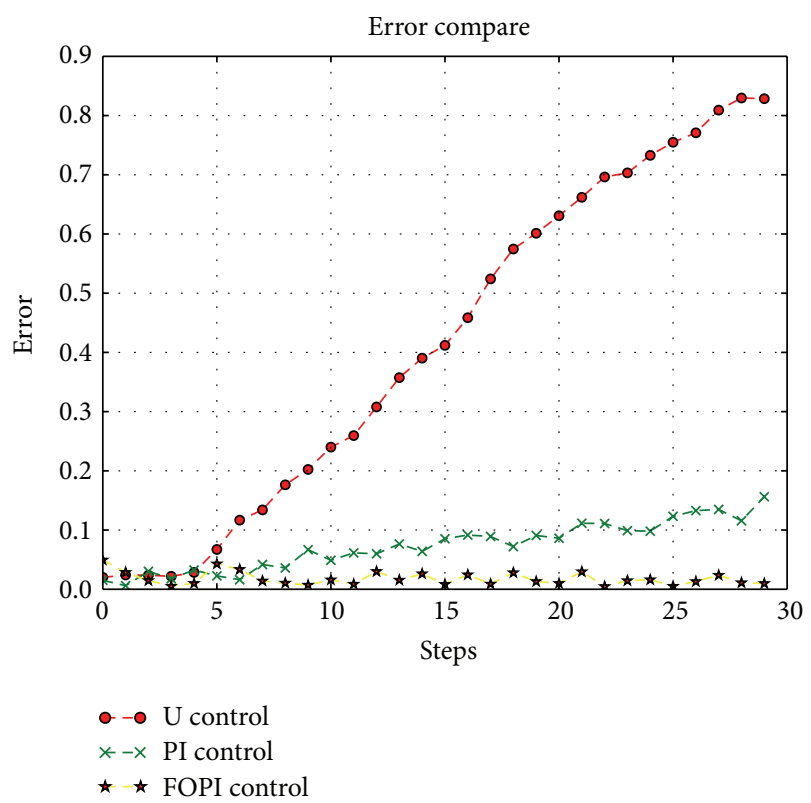

FIGURE 8: The comparison of the error with fixed U/PI/FOPI controller.

\section{Acknowledgments}

The work was partly supported by the National Natural Science Foundation of China (NSFC) under the Project Grant no. 51275439 and the 973 Plan under the Project no. 2013CB733003, and the Natural Science Foundation of Hebei Province of China under the project no. F2014203095.

\section{References}

[1] K. J. Astrom and T. Hagglund, Advanced PID Control, ISA, USA, 2006.
[2] Z. Jiao and Y. Q. Chen, "Stability of fractional-order linear timeinvariant systems with multiple noncommensurate orders," Computers \& Mathematics with Applications, vol. 64, no. 10, pp. 3053-3058, 2012.

[3] R. L. Magin, Fractional Calculus in Bioengineering, Begell House, 2006.

[4] R. Hilfer, Applications of Fractional Calculus in Physics, World Scientific, Singapore, 2000.

[5] I. Petráš, Fractional-Order Nonlinear Systems: Modeling, Analysis and Simulation, Springer, New York, NY, USA, 2011.

[6] F. Mainardi, Fractional Calculus and Waves in Linear Viscoelasticity: An Introduction to Mathematical Models, Imperial College Press, London, UK, 2010.

[7] J. Sabatier, O. P. Agrawal, and J. A. TenreiroMachado, Eds., Advances in Fractional Calculus: Theoretical Developments and Applications in Physics and Engineering, Springer, 2007.

[8] B. M. Vinagre, C. A. Monje, A. J. Caldern, and J. I. Surez, "Fractional PID controllers for industry application: a brief introduction," Journal of Vibration and Control, vol. 13, no. 910, pp. 1419-1429, 2007.

[9] A. J. Calderón, B. M. Vinagre, and V. Feliu, "Fractional order control strategies for power electronic buck converters," Signal Processing, vol. 86, no. 10, pp. 2803-2819, 2006.

[10] M. Schlegel and M. Cech, "Fractal system identification for robust control-the moment approach," in Proceedings of the 5th International Carpathian Control Conference, pp. 1-6, AGHUST, Krakaw, Poland, May 2004.

[11] V. Feliu-Batlle, R. R. Pérez, and L. S. Rodríguez, "Fractional robust control of main irrigation canals with variable dynamic parameters," Control Engineering Practice, vol. 15, no. 6, pp. 673686, 2007.

[12] F. Hongmei, S. Yu, and Z. Xiaobin, "Research on fractional order controller in servo press control system," in Proceedings of the IEEE International Conference on Mechatronics and Automation (ICMA '07), pp. 2934-2938, August 2007.

[13] I. S. Jesus and J. A. Tenreiro Machado, "Fractional control of heat diffusion systems," Nonlinear Dynamics, vol. 54, no. 3, pp. 263-282, 2008.

[14] M. Li, S. C. Lim, C. Cattani, and M. Scalia, "Characteristic roots of a class of fractional oscillators," Advances in High Energy Physics, vol. 2013, Article ID 853925, 7 pages, 2013.

[15] M. Li, "Approximating ideal filters by systems of fractional order," Computational and Mathematical Methods in Medicine, vol. 2012, Article ID 365054, 6 pages, 2012.

[16] M. Li, S. C. Lim, and S. Chen, "Exact solution of impulse response to a class of fractional oscillators and its stability," Mathematical Problems in Engineering, vol. 2011, Article ID 657839, 9 pages, 2011.

[17] H. Hejazi, T. Moroney, and F. Liu, "Stability and convergence of a finite volume method for the space fractional advectiondispersion equation," Journal of Computational and Applied Mathematics, vol. 255, pp. 684-697, 2014.

[18] J. Sabatier and C. Farges, "On stability of commensurate fractional order systems," International Journal of Bifurcation and Chaos, vol. 22, no. 4, Article ID 1250084, 8 pages, 2012.

[19] J.-G. Lu, Y. Chen, and W. Chen, "Robust asymptotical stability of fractional-order linear systems with structured perturbations," Computers \& Mathematics with Applications, vol. 66, no. 5, pp. 873-882, 2013.

[20] I. Podlubny, "Fractional-order systems and $P I^{\lambda} D^{\mu}$-controllers," IEEE Transactions on Automatic Control, vol. 44, no. 1, pp. 208214, 1999. 
[21] I. Podlubny, Fractional Differential Equations, Academic Press, San Diego, Calif, USA, 1999.

[22] Ch. Lubich, "Discretized fractional calculus," SIAM Journal on Mathematical Analysis, vol. 17, no. 3, pp. 704-719, 1986.

[23] I. Petras, "An adaptive fractional-order controller," in Proceedings of the 14th IEEE International Carpathian Control Conference (ICCC '13), pp. 297-2301, Rytro, Poland, May 2013.

[24] I. Podlubny, "Fractional-order systems and fractional order controllers," Technical Report UEF-03-94, Slovak Academy of Sciences. Institute of Experimental Physics, Department of Control Engineering. Faculty of Mining, University of Technology, Kosice, Slovak Republic, 1994.

[25] H. Durrant-Whyte and T. Bailey, "Simultaneous localization and mapping. Part I: the essential algorithms," IEEE Robotics and Automation Magazine, vol. 13, no. 2, pp. 99-108, 2006.

[26] T. Bailey and H. Durrant-Whyte, "Simultaneous localization and mapping (SLAM). Part II: state of the art," IEEE Robotics and Automation Magazine, vol. 13, no. 3, pp. 108-117, 2006.

[27] S. Shamsuddin, L. I. Ismail, H. Yussof et al., "Humanoid robot NAO: review of control and motion exploration," in Proceedings of the IEEE International Conference on Control System, Computing and Engineering (ICCSCE '11), pp. 511-5516, Penang, Malaysia, November 2011. 


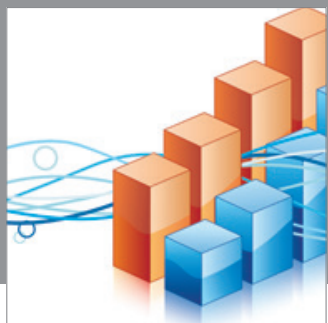

Advances in

Operations Research

mansans

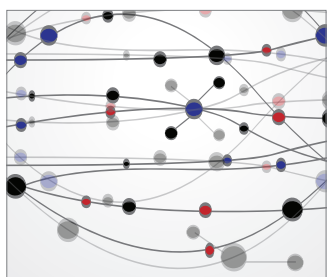

The Scientific World Journal
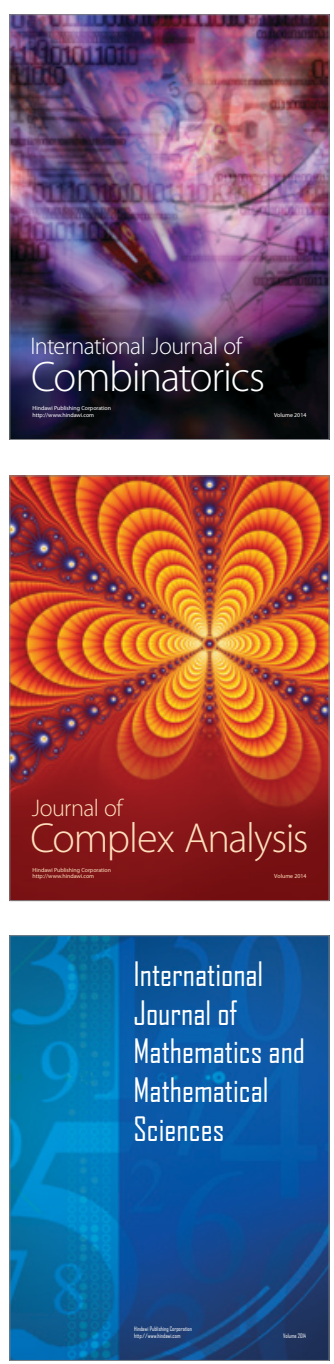
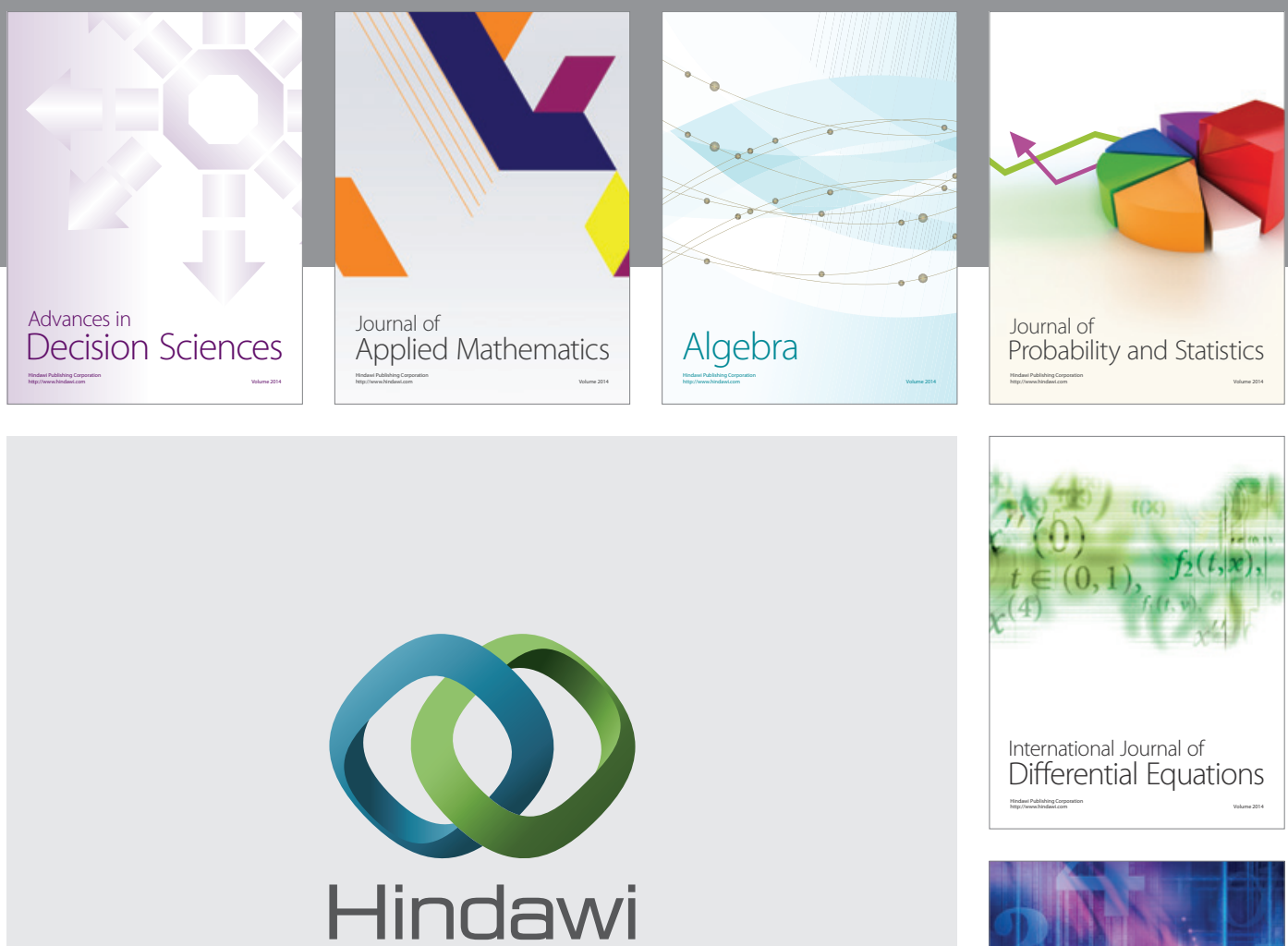

Submit your manuscripts at http://www.hindawi.com
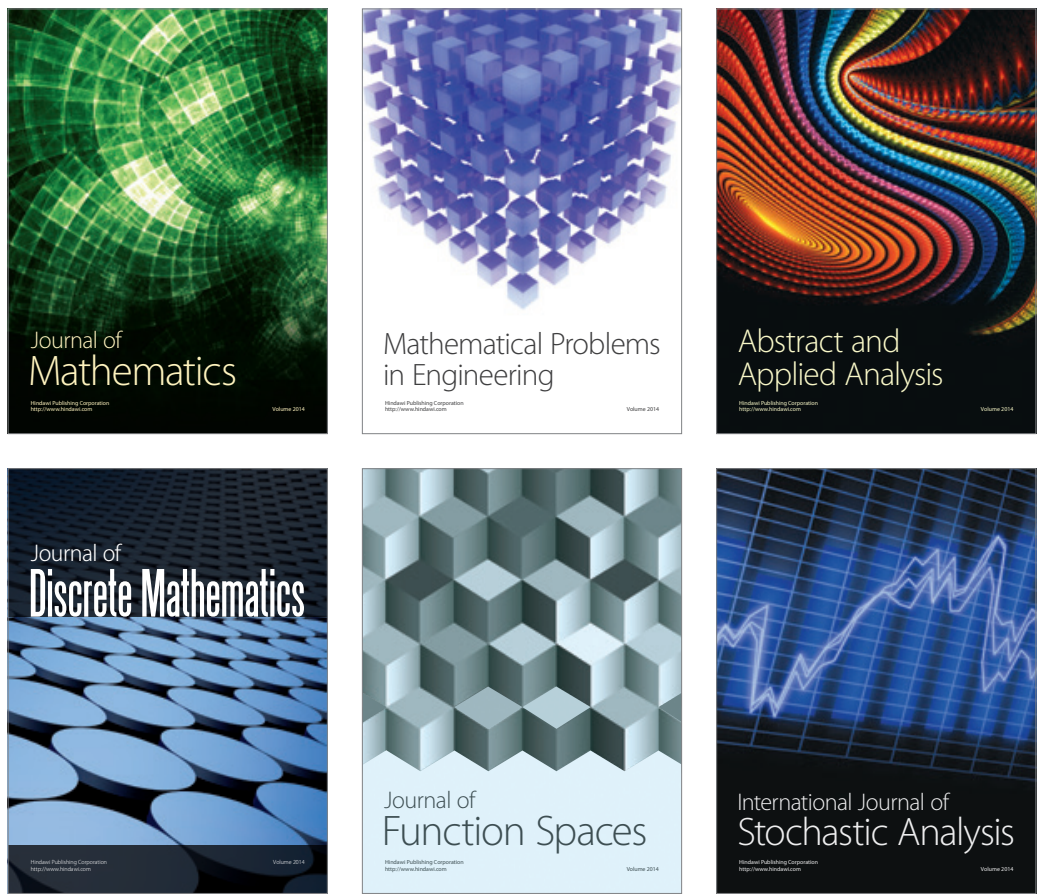

Journal of

Function Spaces

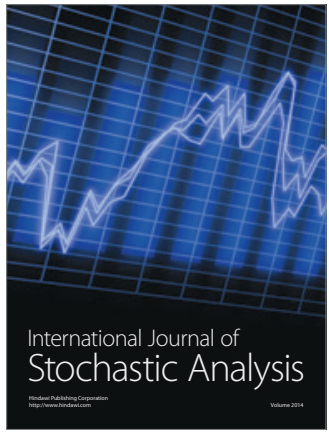

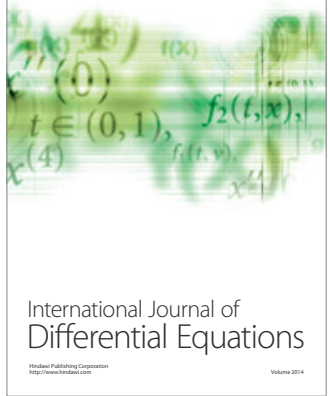
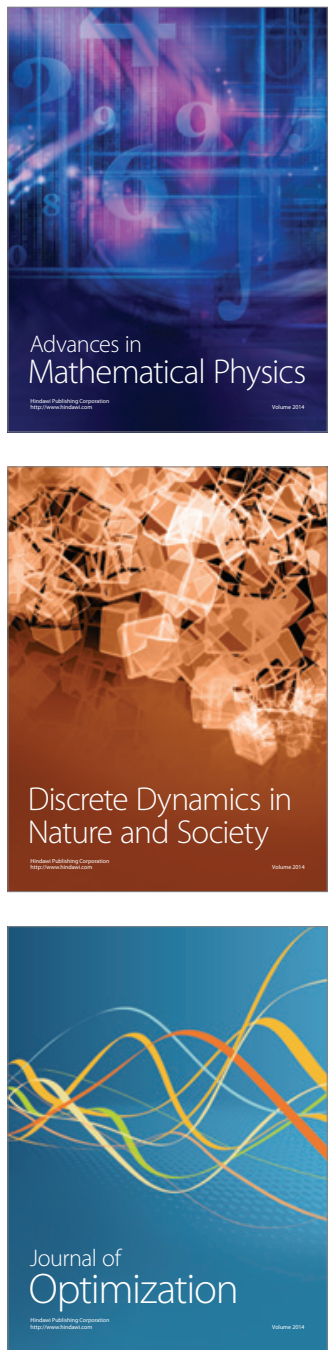